for patients affected by RA and PsA. Based on these promising findings, we aim to extend the approach to seronegative RA as well as early RA and PsA. REFERENCES:

[1] Le, L. et al. (2018). Supervised autoencoders: Improving generalization performance with unsupervised regularizers. In Advances in Neural Information Processing Systems.

[2] Springenberg, J. T. et al. (2015). Striving for simplicity: The all convolutional net. 3rd International Conference on Learning Representations, ICLR 2015 Workshop Track Proceedings.

[3] Simon, D. et al. (2017). Age- and Sex-Dependent Changes of Intra-articular Cortical and Trabecular Bone Structure and the Effects of Rheumatoid Arthritis. Journal of Bone and Mineral Research, 32(4), 722-730.

[4] Folle, L. et al. (2021). Fully Automatic Bone Mineral Density Measurements using Deep Learning. Manuscript submitted for publication.

Acknowledgements: This work was supported by the emerging field initiative (project 4 Med 05 "MIRACLE") of the University Erlangen-Nürnberg and MASCARA - Molecular Assessment of Signatures Characterizing the Remission of Arthritis grant 01EC1903A

Disclosure of Interests: Lukas Folle: None declared, Chang Liu: None declared, David Simon Speakers bureau: Lilly, Novartis, Consultant of: Lilly, Novartis, Gilead, BMS, Abbvie, Grant/research support from: Lilly, Novartis, Timo Meinderink: None declared, Anna-Maria Liphardt Consultant of: Mylan/Meda Pharma, Grant/research support from: Novartis, Gerhard Krönke Speakers bureau: Lilly, Novartis, Consultant of: Lilly, Novartis, Gilead, BMS, Abbvie, Grant/research support from: Lilly, Novartis, Georg Schett Speakers bureau: Lilly, Novartis, Consultant of: Lilly, Novartis, Gilead, BMS, Abbvie, Grant/research support from: Lilly, Novartis, Andreas Maier: None declared, Arnd Kleyer Speakers bureau: Lilly, Novartis, Consultant of: Lilly, Novartis, Gilead, BMS, Abbvie, Grant/research support from: Novartis, Lilly DOI: 10.1136/annrheumdis-2021-eular.383

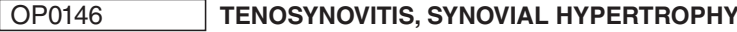 AND FEET BURSITIS ARE USEFUL ULTRASOUND BIOMARKERS FOR PREDICTING ARTHRITIS DEVELOPMENT IN A POPULATION AT-RISK FOR RHEUMATOID ARTHRITIS}

Y. Kisten ${ }^{1}$, A. Circiumaru ${ }^{1}$, M. Loberg ${ }^{1}$, N. Vivar-Pomiano ${ }^{1}$, A. Antovic ${ }^{2}$, H. Rezaei ${ }^{1}$, E. Af Klint ${ }^{1}$, A. Hensvold ${ }^{1,2}$, A. Catrina ${ }^{1,2} .{ }^{1}$ Karolinska Institute, Department of Medicine, Rheumatology Unit and Early Arthritis Clinic of the Karolinska University Hospital, Stockholm, Sweden; ${ }^{2}$ Academic Specialist Center, Center for Rheumatology, Stockholm Health Region, Stockholm, Sweden

Background: Musculoskeletal ultrasound (MSUS) evaluation of individuals at risk for developing rheumatoid arthritis (RA) having Anti-Citrullinated Protein Antibody (ACPA) positivity and musculoskeletal complaints, may play an important role in the very early detection of RA.

Objectives: We aimed to identify which ultrasound markers could predict arthritis development.

Methods: Individuals with musculoskeletal complaints with a positive anti-CCP2 test were referred to the rheumatology department for a detailed clinical (68 joint count) and MSUS examination of the hands, feet and any symptomatic joints. Only those without clinical and/or MSUS detected arthritis were included in the RISK RA prospective cohort and followed-up over 3 years/ or until arthritis onset. Using EULAR-OMERACT guidelines ${ }^{1}$, MSUS markers for synovial hypertrophy ( $\mathrm{SH}$ ) and hyperemia (Doppler activity) were documented for each visit. Finger and wrist tendons were screened for any signs of tenosynovitis (TS), and between metatarsal joints for bursitis. Association of MSUS biomarkers with arthritis development was tested (comparing proportions) using Chi-Squared or Fisher's exact tests.

Results: 288 individuals were included from January 2014 to October 2019 (79\% female, 35\% RF positive, median age 48 years: IQR: 36-58). Within a median of 38 months (IQR: 1-72) since recruitment, 84 individuals (28\%) developed an arthritis diagnosis.

Prior to obtaining any diagnosis (at inclusion and/or follow-up visit), 95 of the 288 individuals (33\%) had at least one type of MSUS anatomical modification present (around the tendons, joint synovium and/or within bursal cavities), and $56 \%$ (53/95) of these individuals eventually developed arthritis. Of the remaining 193 that did not present with any obvious MSUS changes, $16 \%$ progressed towards arthritis development.

The presence of tenosynovitis was detected in 64 of 288 individuals scanned prior to diagnosis and were more frequent in those developing arthritis (44\%, $37 / 84)$ as compared to those with TS not developing arthritis (13\%, 27/204), $p<0.0001$. The extensor carpi ulnaris wrist tendons were mostly involved. Sonographic changes within the synovium were noted in $11 \%(32 / 288)$ of all individuals, mostly affecting the metacarpophalangeal (MCP) and metatarsophalangeal
(MTP) joints. There was a higher incidence of synovial hypertrophy detected in those developing arthritis $(22 \%, 18 / 24)$, as compared to those that remained arthritis free $(7 \%, 14 / 204), p<0.0001$. The MCP joints with synovial hypertrophy were more prone to arthritis development as compared to the MTP's. Further more, we observed a higher frequency of bursitis between the MTP joints in individuals developing arthritis, as compared to individuals having a bursitis who did not develop arthritis $(13 \%, 11 / 84$ versus $7 \%, 14 / 204, p=0.009)$.



Conclusion: Ultrasound biomarkers such as tenosynovitis of the extensor carpi ulnaris, synovial hypertrophy of the MCP joints and feet bursitis have good potential to predict arthritis development in a population at-risk for rheumatoid arthritis. REFERENCES:

[1] Maria-Antonietta D’Agostino et al. RMD Open 2017;3:e 000428

Acknowledgements: All study participants and patients, including researchers that are part of the multidisciplinary laboratory, clinical and academic teams of the RISK RA study group, as well as all assisting this research in one form or the other are greatly acknowledged.

Disclosure of Interests: None declared

DOI: 10.1136/annrheumdis-2021-eular.1676

\section{OP0147 RHEUMATIC? - A DIGITAL DIAGNOSTIC DECISION SUPPORT TOOL FOR INDIVIDUALS SUSPECTING RHEUMATIC DISEASES: A MULTICENTER VALIDATION STUDY}

R. Knevel ${ }^{1}$, J. Knitza ${ }^{2,3,4}$, A. Hensvold ${ }^{5,6}$, A. Circiumaru $^{5,6}$, T. Bruce ${ }^{7}$, S. Evans ${ }^{8}$, T. Maarseveen ${ }^{1}$, M. Maurits ${ }^{1}$, L. Beaart- van de Voorde ${ }^{1}$, D. Simon ${ }^{2,3}$, A. Kleyer ${ }^{2,3}$, M. Johannesson ${ }^{6}$, G. Schett ${ }^{2,3}$, T. Huizinga $^{1}$, S. Svanteson ${ }^{8}$ A. Lindfors ${ }^{7}$, L. Klareskog ${ }^{5}$, A. Catrina ${ }^{5} .{ }^{1}$ LUMC, Rheumatology, Leiden, Netherlands; ${ }^{2}$ Universitätsklinikum Erlangen, Friedrich-Alexander-University Erlangen-Nürnberg (FAU), Department of Internal Medicine 3 - Rheumatology and Immunology, Erlangen, Germany; ${ }^{3}$ FAU Erlangen-Nuremberg and Universitätsklinikum, Deutsches Zentrum fuer Immuntherapie (DZI), Erlangen, Germany; ${ }^{4}$ Université Grenoble Alpe, AGEIS, Grenoble, France; ${ }^{5}$ Karolinska Institutet, Karolinska University Hospital, Division of Rheumatology, Department of Medicine, Stockholm, Sweden; ${ }^{6}$ Academic Specialist Center, Center for Rheumatology, Center for Rheumatology, Stockholm Region, Sweden; ${ }^{7}$ Ocean Observations, a Design Consultancy, Stockholm, Sweden; ${ }^{8}$ Elsa Science, A Digital Health Company, Stockholm, Sweden

Background: Digital diagnostic decision support tools promise to accelerate diagnosis and increase health care efficiency in rheumatology. Rheumatic? is an online tool developed by specialists in rheumatology and general medicine together with patients and patient organizations for individuals suspecting a rheumatic disease. ${ }^{1,2}$ The tool can be used by people suspicious for rheumatic diseases resulting in individual advise on eventually seeking further health care Objectives: We tested Rheumatic? for its ability to differentiate symptoms from immune-mediated diseases from other rheumatic and musculoskeletal com plaints and disorders in patients visiting rheumatology clinics. 